\title{
Comparison of Bone Marrow Transplantation and Chemotherapy for Acute Leukemia
}

Bone marrow transplantation (BMT) in Japan was started in 1975.

At present, a total of 1,525 patients are registered to receive BMT. In 1990, 354 patients including 164 with acute leukemia received BMT. The long-term survival rate in early leukemia in the Japanese registry has risen to $60 \%$. The results of chemotherapy (CT) for acute leukemia have also shown remarkable improvement. Ohno (1) reported a value of $43 \%$ for a 3.5 -year survival in the Japan Acute Leukemia Study Group (JALSG) protocol.

Regarding a comparison of both modes of treatment of acute leukemia, many reports show varying results between studies.

Appelbaum et al (2) reported significant differences in the survival between patients treated with BMT and CT, while Zittoun et al (3) reported no difference between the two groups of patients. There appears to be many factors in the comparison of BMT and CT which need careful consideration.

\section{Subjects of comparison}

BMT is fundamentally a supportive treatment in intensive therapy to overcome marrow toxicity. In this sense it has many similarities to blood transfusion. A comparison of BMT with $\mathrm{CT}$ resembles a comparison between blood transfusion and $\mathrm{CT}$, although the latter may appear somewhat ridiculous at the present time.

\section{Patient comparison}

For example if we compare the data of BMT registry with those obtained from a large scale CT protocol study, there are biases in the patients studied, because the registry includes all patients and the protocol study selects the patients fitted for the protocol.

A randomized prospective study is considered to be the best testing method at present. However, as both BMT and CT are changing very rapidly with the new drugs or new cytokines, it is difficult to keep a fixed regimen for a large-scale study requiring a 5-year follow up.

\section{Purpose}

If we want to use the results of a study to select treatment for a certain patient who will receive treatment at one center, the comparison of BMT and CT in that center seems the most appropriate for such a consideration. For a comparison of two $\mathrm{CT}$ regimens with and without BMT a prospective randomized controlled study seems to give the most reliable data for the regimens tested. For the comparison of BMT in general with $\mathrm{CT}$ in general, data of large-scale registry for both BMT and CT are necessary to obtain an overview of both treatments. From the above consideration the following types of study are required.

1. Comparison of BMT and $\mathrm{CT}$ in each center This will be helpful for the selection of BMT or CT for the patient who will be treated at one center, or for $\mathrm{CT}$ data in center $\mathrm{A}$ and $\mathrm{BMT}$ data in center $\mathrm{B}$ for a patient who will receive $C T$ at center $A$ and $B M T$ at center B. The data of each center seems the most important and most useful data. We think each center should prepare the data of their patients.

2. Comparison of BMT and CT under certain regimens in a cooperative randomized controlled study

This may provide an evaluation of BMT and CT under a certain regimen.

3. Comparison of registry data of BMT and CT

This may give an overview of BMT and CT in general.

Future changes

The survival of patients treated with $\mathrm{CT}$ has been improved by dose elevation or shortening of the interval. CT has been improved by enhancing intensity or toxicity. It is highly expected that $\mathrm{CT}$ will be more intensive or more toxic in the future. BMT has also shown remarkable progress in recent years.

Successful prophylaxis and treatment of the major causes of death such as interstitial pneumonitis, graft versus host disease (GVHD), rejection and infection have decreased the failure of BMT and elevated the rate of long-term survival. BMT has been improved by enhancing safety. It is also clearly expected that BMT will be safer in the near future. As stated previously, the comparison between blood transfusion and CT seems a somewhat ridiculous comparison.

At present blood transfusion has become a quite safe supportive treatment. No doctor is worried about giving blood transfusion when necessary. It is now an indispensable routine support for CT. Therefore, it is no longer possible to set an arm of CT without blood transfusion in a comparative study.

If BMT becomes sufficiently safe and CT becomes sufficiently intensive or toxic in the future, we consider that all patients who receive intensive CT will be supported by either allogeneic or autologous BMT. BMT will be safe routine supportive treatment for CT. Then 
it may be no longer possible to set an arm of $\mathrm{CT}$ without BMT in the comparative study. We consider that the discussion of comparing BMT with CT will no longer occur at that stage.

One of the limiting factors in this last step may be the cost benefit of BMT. Hiraoka (4) has reported that the medical cost in Japan of CT for one more year of survival in patients with acute leukemia is 4.8 million yen, whereas it is 3.8 million yen for BMT. Medical cost also seems to be one of the important endpoints in the comparison of BMT and CT.

The comparison of BMT and CT for acute leukemia seems to involve many factors which need to be studied at present and adjusted according to the progress of technology in the near future.
Tohru Masaoka, M.D. Center for Adult Diseases, 1-3-3 Higashinariku Nakamichi 537 Osaka, Japan

\section{References}

1) Ohno R. Recent progress in the treatment of adult acute leukemia. Acta Heamatologica Jpn 52: 1287, 1989.

2) Appelbaum FR, Dahlberg S, Thomas ED, et al. Bone marrow transplantation or chemotherapy after remission induction for adults with acute non-lymphoblastic leukemia. A prospective comparison. Ann Intern Med 101: 581, 1984.

3) Zittoun R, Jehn U, Fiere D, et al. Alternating $\mathrm{v}$ repeated postremission treatment in adult acute myelogenous leukemia randomized Phase II study (AML6) of the EORTC leukemia. Blood 73: 896, 1989.

4) Hiraoka A, Masaoka T. Cost effectiveness of allogeneic bone marrow transplantation for acute leukemia. Rinsyokagaku 28: 849, 1992 (in Japanese). 